\title{
Cross-Layering between Physical Layer and Routing in Wireless Ad-Hoc Networks
}

\author{
Jean Michel Dricot ${ }^{1}$, Gianluigi Ferrari ${ }^{2}$, and Philippe De Doncker ${ }^{1}$ \\ 1 Université Libre de Bruxelles, OPERA Department \\ Wireless Communications Group \\ 1050 Bruxelles, Belgium \\ \{jdricot, pdedonck\}@ulb.ac.be \\ 2 University of Parma, Department of Information Engineering \\ Wireless Ad-hoc and Sensor Networks (WASN) Lab \\ 43124 Parma, Italy \\ gianluigi.ferrari@unipr.it
}

\begin{abstract}
Routing is a key issue in wireless ad-hoc networks. The goal of an efficient routing strategy is to set up routes so that the overall quality of communications will be the best possible. While the Open Systems Interconnection (OSI) reference model advocates for a clear separation of routing, access, and physical layers, in this paper we show that in scenarios with faded communications, cross-layer interactions have to be carefully considered. More precisely, we compare the performance of the Ad-hoc On-demand Distance Vector (AODV) routing algorithm with that of one of its physical layer-oriented variants, denoted as $\operatorname{AODV} \varphi$. It will be clearly shown that no single routing strategy is always optimal and that an intelligent adaptation should be performed.
\end{abstract}

Keywords: Wireless networks, sensor networks, cross-layer, channel modeling, performance evaluation, fading.

\section{Introduction}

A wireless ad-hoc network consists of a large number of autonomous mobile nodes connected to each other directly and without the need for pre-existing configuration parameters or centralized infrastructures 1,2. From an architectural point of view, a Mobile Ad-Hoc Network (MANET) is formed as a multi-hop architecture due to the limited transmission range of wireless transceivers and node mobility. Therefore, routing plays an important role in the operation of such networks: each node has to act as both router and host.

While the Open Systems Interconnection (OSI) reference model advocates for a clear separation of the routing, access, and physical layers, this distinction is seldom guaranteed in several future generation wireless network architectures [3]. In fact, the physical layer is very variable and its characteristics profoundly influences the performance of MANETs. Therefore, its interaction with the upper layers often leads to unexpected and undesirable effects. This paper analyzes the existing cross-layer interactions between the physical layer and the routing strategy.

D. Ślęzak et al. (Eds.): FGCN/ACN 2009, CCIS 56, pp. 324-333, 2009.

(C) Springer-Verlag Berlin Heidelberg 2009 
The goal of a routing algorithm is to find the best route according to a proper cost function. For instance, possible strategies include shortest-path routing [4, energy-aware routing [5, 6, 7, highest stability routing 8, or least-congested route selection 8]. Meanwhile, these approaches typically do not take into account the cross-layer interactions suggested in [9, 10, 3, which clearly highlight the impact that physical layer has on routing efficiency. In particular, the bit error rate (BER) at the end of a multi-hop route may, under certain conditions, represent a good indicator of the physical layer status [11,12]. Also, it has been recently shown that the expected transmission (ETX) count of a path, defined as the expected total number of packet transmissions (including retransmissions) required to successfully deliver a packet along that path, is a good indicator of the routing quality [13. The impact of the cross-layer interactions has received a significant attention in the last years. The interaction between the physical and the application layers (and, more precisely, VoIP and video throughput) has been quantified in [14. The impact of the interference on throughput 15] and connectivity [16] has also been highlighted.

In the following, we consider a regular network topology, in the sense that every receiver has the same number of nearest neighbors and the same distance to any of these nearest neighbors. In reality, the distances will more likely be non-homogeneous and distributed around an average value. The impact of the topology is out of the scope of this paper and can be found in [17. Also, we consider a simple slotted asynchronous random access scheme, such that, in each timeslot, every node transmits with a given probability $q$ (obviously proportional to the traffic load). The assumption of a simple Bernoulli transmission model, which is supported by the analyses presented in [18 and [19, p. 278] can be considered simpler than other channelization schemes (such as time/frequency division multiple access) but it will allow to derive a lower bound on the performance of communication networks with more sophisticated MAC protocols under use.

\section{The Log-Normal Fading Link Model}

We assume a narrowband log-normal block fading (i.e., shadowing or slow fading) channel [20]. This model is implemented in the NS-2 simulator and will be used later for a simulation-based investigation of cross-layer interactions. In the log-normal fading model, the ratio of transmit-to-receive power $X \triangleq P_{t} / P_{0}$ is assumed to have the following log-normal distribution:

$$
f_{X}(x)=\frac{1}{x \sqrt{2 \pi \sigma^{2}}} \exp \left[-\frac{(\ln x-\mu)^{2}}{2 \sigma^{2}}\right] U(x) .
$$

where $\mu$ is the path loss attenuation (dimension: $[\mathrm{dB}]$ ) that can be based on an analytical model or empirical measurements and $\sigma$ is the standard deviation of $X$ (dimension: $[\mathrm{dB}]$ ). Most empirical studies support a standard deviation $\sigma=4-16 \mathrm{~dB}$. Alternatively (and in the model implemented by the NS-2), the 
path loss can be treated separately from the slow fading by letting $\mu=0$ and setting

$$
P_{0}=P_{\mathrm{t}} d^{-n} X
$$

where $P_{0}$ is the received power (dimension: [W]), $P_{\mathrm{t}}$ is the transmit power (dimension: $[\mathrm{W}]), n$ is the path loss exponent (adimensional). A transmission on the considered link is successful if and only if the signal-to-noise and interference ratio (SINR) at the receiver, denoted as SINR, is above a pre-defined threshold $\Theta$. This threshold value depends, among other factors, on the receiver characteristics, the modulation and coding scheme, etc. 21]. The SINR can then be written as $\mathrm{SINR} \triangleq P_{0} / N+P_{\text {int }}$ where $N$ is the background noise power and $P_{\text {int }}$ is the total interference power at the receiver, given by the sum of the received powers from all the undesired transmitters. In large and/or dense networks, the transmission is only limited by the interference and it can be assumed that $N \ll P_{\text {int }}$. The total interference in a scenario with $j$ interfering neighbors is $P_{\text {int }} \triangleq \sum_{i=1}^{j} P_{i}=\sum_{i=1}^{j} P_{\mathrm{c}} d^{-n} X_{i}$ where $\left\{X_{i}\right\}_{i=1 \ldots j}$ are log-normally distributed and represent the independent fading processes associated with the links originating at the interfering nodes. The probability of successful link transmission in the presence of $j$ interferers is

$$
\mathcal{P}_{\mathrm{s}}^{(j)} \triangleq \mathbb{P}\{\operatorname{SINR}>\Theta \mid j \text { interferers }\}=\mathbb{P}\left\{\frac{P_{0} d_{0}^{-n} X}{\sum_{i=1}^{j} P_{c} d^{-n} X_{i}}>\Theta\right\} .
$$

By introducing the new r.v. $Y^{(j)} \triangleq \sum_{i=1}^{j} X_{i}$, and $\xi \triangleq \Theta \frac{P_{\mathrm{c}}}{P_{0}}\left(\frac{d_{0}}{d}\right)^{n}$ it follows:

$$
\mathcal{P}_{\mathrm{s}}^{(j)}=1-\mathbb{P}\left\{X \leq \xi Y^{(j)}\right\}=1-\iint_{x \leq \xi y} f_{X Y^{(j)}}(x, y) \mathrm{d} x \mathrm{~d} y
$$

where $f_{X Y^{(j)}}(x, y)$ is the joint probability density function of $X$ and $Y^{(j)}$. Since $X$ and $Y^{(j)}$ are independent, i.e., $f_{X Y^{(j)}}(x, y)=f_{X}(x) f_{Y^{(j)}}(y)$. In the case of the log-normal fading, $Y^{(j)}$ corresponds to the sum of log-normal random variables with the same parameters. Following the approach proposed in [22,23], $Y^{(j)}$ can be approximated as a log-normal random variable with parameters $\sigma_{j}^{2}=\ln \left(\frac{e^{\sigma^{2}}-1}{j}+1\right), \mu_{j}=\ln j+\frac{\sigma^{2}-\sigma_{j}^{2}}{2}$. Finally, the probability of successful link transmission becomes

$$
\mathcal{P}_{\mathrm{S}}^{(j)}=\frac{1}{2}-\frac{1}{2} \int_{0}^{\infty} \frac{\exp \left[-\frac{\left(\ln y-\mu_{j}\right)^{2}}{2 \sigma_{j}^{2}}\right]}{y \sigma_{j} \sqrt{2 \pi}} \operatorname{erf}\left(\frac{\ln \xi y}{\sigma \sqrt{2}}\right) \mathrm{d} y .
$$

A closed-form expression of $\mathcal{P}_{\mathrm{S}}^{(j)}$ cannot be derived and its integral expression must be numerically evaluated. In Fig. 1. $\mathcal{P}_{\mathrm{s}}^{(j)}$ is shown, as a function of the log-normal fading power $\sigma$, for various values of the number of interferers $j$. In all cases, $\xi=10 \mathrm{~dB}$, and this corresponds to keeping the SINR threshold fixed. The figure suggests that the presence of lognormal shadowing improves 


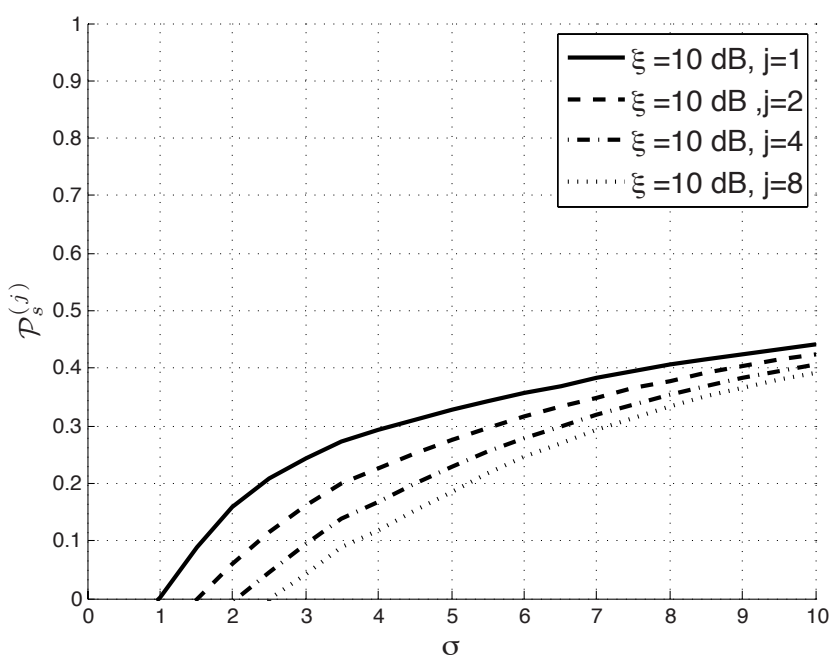

Fig. 1. Probability of link success $\operatorname{Pr}_{\mathrm{s}}^{(j)}$ as a function of $\sigma$ and for a fixed value of $\xi$. Various values of the number of active interferers $j$ are considered.

the connectivity properties of the network, as predicted by the results in 24, 25]. Furthermore, the link probability of success is monotonically increasing in the lognormal spread $\sigma$ so that it can be concluded that, even though the presence of a deep fades impacts the received power on the link, it has globally a beneficial impact on the link SINR.

Let us now considerer a link surrounded by $N$ possible interfering neighbors, each of them having a probability $q$ of transmitting a packet at a given time. In that case, the total interference is: $P_{\text {int }} \triangleq \sum_{i=1}^{N} P_{i} \Lambda_{i}$ where $\left\{\Lambda_{i}\right\}_{i=1 \ldots N}$ is a sequence of stochastically independent Bernoulli distributed random variables with $\mathbb{P}\left\{\Lambda_{i}=1\right\}=q$ and $\mathbb{P}\left\{\Lambda_{i}=0\right\}=1-q$. The probability that $j$ nodes among the $N$ nodes interfere at the same moment is thus a binomial r.v. with parameters $q$ and $N: \mathbb{P}\{j$ interferers $\mid q, N\}=\left(\begin{array}{c}N \\ j\end{array}\right) q^{j}(1-q)^{N-j}$ and the total probability of link success in the presence of $N$ neighbors is

$$
\begin{aligned}
\mathcal{P}_{\mathrm{s}} \triangleq \mathbb{P}\{\operatorname{SINR}>\Theta\} & =\sum_{j=0}^{N} \underbrace{\mathbb{P}\{\operatorname{SINR}>\Theta \mid j \text { interferers }\}}_{\mathcal{P}_{\mathrm{s}}^{(j)}} \cdot \underbrace{\mathbb{P}\{j \text { interferers }\}}_{\left(\begin{array}{c}
N \\
j
\end{array}\right) q^{j}(1-q)^{N-j}} \\
& =\sum_{j=0}^{N}\left(\begin{array}{c}
N \\
j
\end{array}\right) \mathcal{P}_{\mathrm{s}}^{(j)} q^{j}(1-q)^{N-j} .
\end{aligned}
$$

where $\mathcal{P}_{\mathrm{s}}^{(j)}$ is given by (2). In Fig. 2] the link probability of success is presented as a function of the amount of active interfering nodes $N$ and the probability of transmission $q$. The variable $\xi$ is set to $10 \mathrm{~dB}$. It can be observed that, even if the amount of interferers increases, the link probability of success decreases to a 


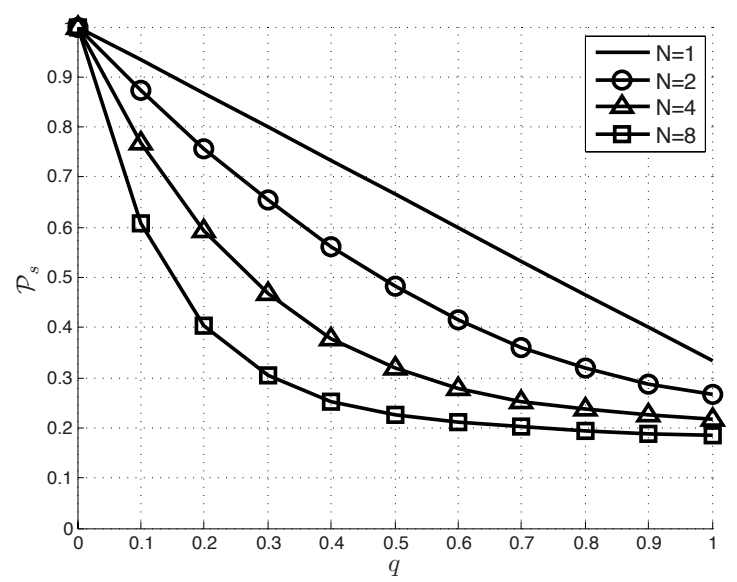

Fig. 2. Link probability success on a single link as a function of the probability of transmission $q$ and the amount of neighbors $N$. The value of $\xi=10 \mathrm{~dB}$ and $\sigma=10 \mathrm{~dB}$.

non-zero value. This is unlike in the fast fading (i.e., Rayleigh fading) scenario where the link probability of success rapidly decreases to $\mathcal{P}_{\mathrm{s}}=0$ as $q$ increases [17.

\section{Simulation-Based Analysis}

Section 4 will present the key results of a simulation-based analysis of the crosslayer interactions between the physical layer and the routing. In order to perform this analysis, we compaired the Ad-hoc On-demand Distance Vector (AODV) routing algorithm and on one of its possible physical layer-oriented variants, denoted as $\operatorname{AODV} \varphi$.

The AODV routing protocol belongs to the class of on-demand routing strategies 26, 27]: a route is created at the time a source needs to reach a destination. In order to locate the destination node, the source broadcasts a route request (RREQ) message all over the network. Each time an intermediate node is solicited, it adds an entry in its routing tables and builds a reverse path to the source. This flooding operation stops when the messages reach the destination or when a node has already the destination in its routing table. A unicast route reply (RREP) message is sent along the reverse path leading to the source. At that moment, the route is formed and kept in cache. When a link breaks, due to mobility or bad propagation conditions, a new route discovery is triggered [28]. Finally, for each destination the route lengths are regularly computed and only the shortest route is kept, i.e., the one with smallest hop count.

The AODV $\varphi$ routing algorithm is a variant of the AODV routing protocol. It implements the relaying strategy by selecting the closest possible neighbour as the next hop when constructing the routes. Only the RREQ messages of AODV are suitably modified and the remaining of the AODV protocol is kept as-is. 
Table 1. Reference values for the parameters used in the simulations

\begin{tabular}{|c|c|}
\hline Number of nodes $N$ & 50 \\
\hline Area $A[\mathrm{~m} \times \mathrm{m}]$ & $1500 \times 300$ \\
\hline Node spatial density $\rho_{\mathrm{S}}\left[\mathrm{m}^{-2}\right]$ & $1.1 \times 10^{-4}$ \\
\hline Active source nodes $N_{\mathrm{a}}$ & 10 \\
\hline MAC Protocol & DCF (IEEE 802.11$)$ \\
\hline Attenuation model & Shadowing \\
\hline Bit rate $R_{\mathrm{b}}[\mathrm{Mb} / \mathrm{s}]$ & 2 \\
\hline Carrier frequency $f_{\mathrm{c}}[\mathrm{MHz}]$ & 914 \\
\hline Max. transmit power $P_{\mathrm{t}}^{\mathrm{max}}[\mathrm{W}]$ & 0.282 \\
\hline Initial node energy $[\mathrm{J}]$ & 30 \\
\hline Send buffer $[\mathrm{pck}]$ & 64 \\
\hline Interface queue $[\mathrm{pck}]$ & 64 \\
\hline Source type & 0.1 \\
\hline Probability of transmission $q$ & $3.652 \times 10^{-10}$ \\
\hline Correct receive threshold $[\mathrm{W}]$ & $1.559 \times 10^{-11}$ \\
\hline Threshold to avoid collisions $[\mathrm{W}]$ & 10 \\
\hline Collision Threshold $\Theta[\mathrm{dB}]$ & 6000 \\
\hline Simulation time $[\mathrm{s}]$ & 600 \\
\hline Pause time $[\mathrm{s}]$ & 1 \\
\hline Node speed $v[\mathrm{~m} / \mathrm{s}]$ & \\
\hline
\end{tabular}

Finally, two performance metrics have been chosen in order to evaluate the performance of the routing protocols of interest: (i) the route throughput (also referred to as packet delivery fraction) that is is defined as the ratio between the packets that successfully reach their destinations and the total number of generated packets, and (ii) the normalized routing load that is the ratio between the number of routing messages and the number of correctly delivered packets. The other reference values for the simulation parameters are presented in Table 1 .

\section{Interaction between Physical Layer and Routing Protocol}

\subsection{Route Throughput}

In Fig. 3 the throughput is shown as a function of the fading spread $\sigma$, for two possible values of $n$ and considering both the $\operatorname{AODV}$ and $\operatorname{AODV} \varphi$ protocols. It can be observed that, when the fading power increases, the performance of the AODV protocol, in terms of route throughput, decreases significantly. This is an undesirable side effect of the longest hop routing strategy: in fact, when the hops are as long as the maximum transmission distance, the received power is the lowest possible. Therefore, in the presence of strong fading, it is more likely that the link SINR will fall under the minimum threshold value and an outage will occur. On the other hand, by combining expressions (1) and the definition 


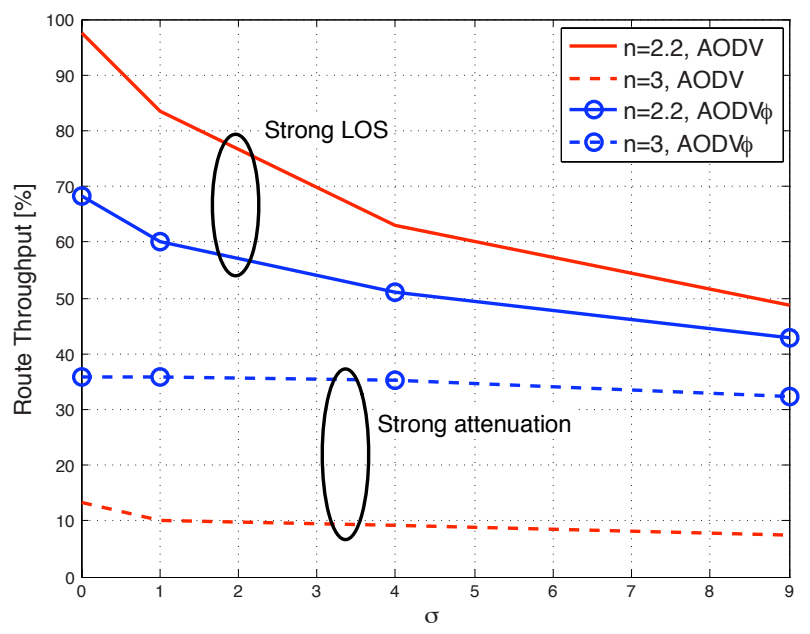

Fig. 3. Route throughput as a function of the fading spread, considering various values of the pathloss exponent $n$ and the two routing strategies of interest

of the SINR, one can see that since the $\operatorname{AODV} \varphi$ protocol chooses shorter hop lengths than the AODV protocol, the corresponding SINR will be significantly higher and the outage event will occur less likely. From the results in Fig. 3, it can also be observed that, when the path loss exponent is high, the fading power (represented by the spread $\sigma$ ) plays little or no role.

It is interesting to note that an alternative analysis of the impact of the fading is carried out in [17] in the case of small-scale block fading (or Rayleigh fading). The authors find that the expression of the probability of successful link transmission depends only on (i) the path loss coefficient, (ii) the transmit power, and (iii) the network topology. Therefore, one can conclude that crosslayering between physical layer and routing protocol is limited to scenarios with large-scale fading (and not in scenarios with Rayleigh fading only).

\subsection{Normalized Routing Load}

In Fig. 4, the normalized routing load is shown as a function of the fading spread, considering the two routing strategies of interest and two possible values for the pathloss exponent $n$. It can be seen that, in the case of a limited attenuation (strong line-of-sight), the normalized routing load required by the AODV $\varphi$ protocol is high since the routes contain a significant number of relaying nodes and this requires a substantial amount of control messages to create and maintain them. When the attenuation increases, the normalized routing load required by the AODV protocol increases significantly and exceeds the load observed with $\operatorname{AODV} \varphi$ protocol. This is due to the extended amount of route repairs triggered by the fragile long hops selected by the AODV protocol and the increasing lengths of the routes. Note that a positive feature of the AODV $\varphi$ protocol 


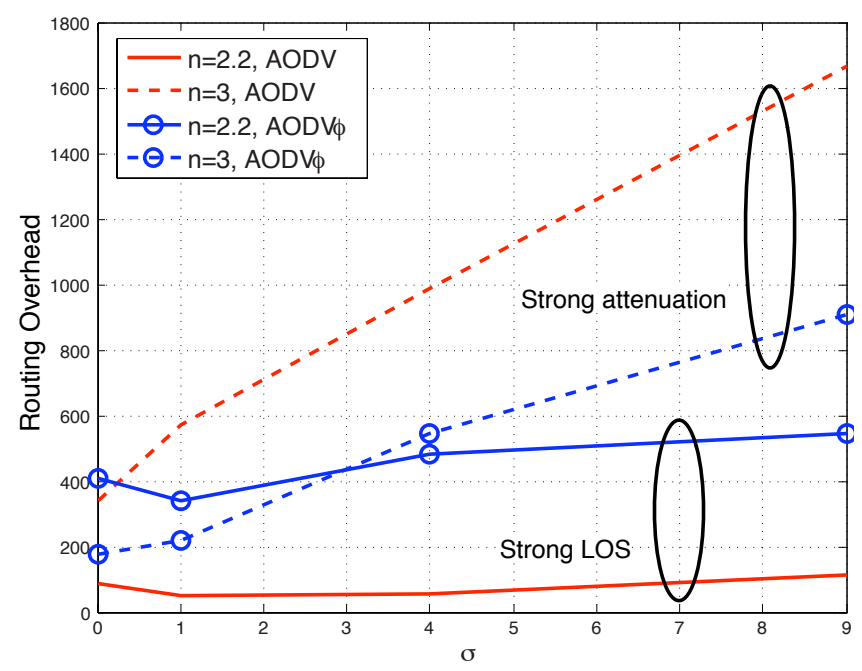

Fig. 4. Normalized routing load as a function of the fading spread, considering various values of the pathloss exponent $n$ and the two routing strategies of interest

consists of the fact that the number of control messages observed in presence of attenuation does not extensively diverge from the strong line-of-sight situation.

Node mobility has also a clear impact of the performance of the two routing protocols (note that, according to the simulation parameters shown in Table 1 . the pause time is one tenth of the simulation time). Indeed, when the attenuation is high (i.e., when the transmission distance is low) the probability that a nodes leaves the coverage zone of an emitter is important, which explains the poor performance of AODV in presence of strong attenuation. On the other hand, in the initial phase of the $\operatorname{AODV} \varphi$ protocol, the closest neighbours is picked up as the next hop. Therefore, it remains longer in the connectivity zone. The rate at which route reconstructions is triggered remains low.

\section{Conclusions}

In this paper, we have investigated the possible cross-layer interactions between the physical layer, medium access control, and the routing strategy. First, we have shown that for a noiseless wireless ad-hoc network, it is reasonable to adapt the routing strategy with respect to the propagation conditions. More precisely, a shortest-hops strategy is preferred when the attenuation raises above a certain level. This strategy has shown to be less sensitive to the intensity of the fading. On the opposite, classical approaches (i.e., the AODV protocol) give better results when the attenuation is limited. Recent work has shown that a reasonable estimation for the value of the attenuation factor can be easily measured by the nodes 29]. Therefore, the routing strategy should be selected according to the detected value of the attenuation factor. 


\section{Acknowledgment}

This work is supported in part by the Belgian National Fund for Scientific Research (FRS-FNRS).

\section{References}

1. Boukerche, A.: Algorithms and Protocols for Wireless, Mobile Ad Hoc Networks. Wiley-IEEE Press (2008)

2. Ramanathan, R.: Challenges: a radically new architecture for next generation mobile ad hoc networks. In: Proc. of the 11th annual international conference on Mobile computing and networking (MobiCom 2005), Cologne, Germany, September 2005, pp. 132-139 (2005)

3. Choi, J., Park, K., Kim, C.-k.: Analysis of cross-layer interaction in multirate 802.11 wlans. IEEE Transactions on Mobile Computing 8(5), 682-693 (2009)

4. Tan, K., Zhang, Q., Zhu, W.: Shortest path routing in partially connected ad hoc networks. In: Proc. of the IEEE Global Telecommunications Conference (GLOBECOM 2003), San Francisco, CA, USA, December 2003, vol. 2, pp. 1038-1042 (2003)

5. Dietrich, I., Dressler, F.: On the lifetime of wireless sensor networks. ACM Trans. Sen. Netw. 5(1), 1-39 (2009)

6. Park, G., Lee, S.: A routing protocol for extend network lifetime through the residual battery and link stability in manet. In: ACC 2008: Proceedings of the WSEAS International Conference on Applied Computing Conference, pp. 199-204. World Scientific and Engineering Academy and Society (WSEAS), Wisconsin (2008)

7. Mohanoor, A.B., Radhakrishnan, S., Sarangan, V.: Online energy aware routing in wireless networks. Ad Hoc Netw. 7(5), 918-931 (2009)

8. Meng, L., Wu, W.: Dynamic source routing protocol based on link stability arithmetic. In: ISISE 2008: Proceedings of the 2008 International Symposium on Information Science and Engieering, pp. 730-733. IEEE Computer Society, Washington (2008)

9. Akyildiz, I.F., Wang, X.: Cross layer design in wireless mesh networks. IEEE Transactions on Vehicular Technology 57(2) (2008)

10. Dricot, J.-M., De Doncker, P., Zimányi, E.: Multivariate analysis of the cross-layer interaction in wireless network similulations. In: Proc. of the Int. Workshop on Wireless Ad-hoc Networks (IWWAN 2005), London, United Kingdom (May 2005)

11. Ferrari, G., Malvassori, S.A., Tonguz, O.K.: On physical layer-oriented routing with power control in ad-hoc wireless networks. IET Communications 2(2), 306319 (2008)

12. Tonguz, O.K., Ferrari, G.: Ad Hoc Wireless Networks: A Communication-Theoretic Perspective. John Wiley and Sons, Chichester (2006)

13. De Couto, D.S.J.: High-throughput routing for multi-hop wireless networks. Ph.D. dissertation, supervisor-Morris, Robert T (2004)

14. Tobagi, F.A., Vyas, A.K., Ha, S., Awoniyi, O.: Interactions between the physical layer and upper layers in wireless networks. Ad Hoc Networks, 1208-1219 (2007)

15. Vyas, A., Tobagi, F.: Impact of interference on the throughput of a multihop path in a wireless network. In: Proceeding of the IEEE third International Conference on Broadband Communications, Networks and Systems, BROADNETS (2006) 
16. Dousse, O., Baccelli, F., Thiran, P.: Impact of interferences on connectivity in ad hoc networks. IEEE/ACM Trans. Netw. 13(2), 425-436 (2005)

17. Liu, X., Haenggi, M.: Throughput Analysis of Random and Regular Networks. EURASIP Journal on Wireless Communications and Networking 4, 554-564 (2005)

18. Tobagi, F.: Analysis of a two-hop centralized packet radio network-part i: slotted aloha. IEEE Trans. Commun. 28(2), 196-207 (1980)

19. Bertsekas, D., Gallager, R.: Data networks, 2nd edn., p. 278. Prentice-Hall, Inc., Upper Saddle River (1992)

20. Catedra, M.F., Perez, J.: Cell Planning for Wireless Communications. Artech House, Inc., Norwood (1999)

21. Rappaport, T.S.: Wireless Communications: Principles and Practice. IEEE Press, Piscataway (1996)

22. Felton, L.F.: The sum of log-normal probability distributions in scatter transmission systems. IRE Trans. on Communications Systems, 57-67 (March 1960)

23. Schwartz, S.C., Yeh, Y.S.: On the distribution function and moments of power sums with log-normal components, vol. 61, pp. 1441-1462 (September 1982)

24. Hekmat, R., Van Mieghem, P.: Connectivity in wireless ad-hoc networks with a log-normal radio model. Mob. Netw. Appl. 11(3), 351-360 (2006)

25. Miorandi, D., Altman, E., Alfano, G.: The impact of channel randomness on coverage and connectivity of ad hoc and sensor networks. IEEE Trans. Wireless Comms. (2007)

26. Perkins, C.: Ad-hoc on-demand distance vector routing (1997)

27. Belding-Royer, E.: Routing approaches in mobile ad hoc networks. In: Ad Hoc Networking. IEEE Press, Los Alamitos (2003)

28. Perkins, C.E., Royer, E.M.: Ad-hoc on-demand distance vector routing. In: Proc. of the Second IEEE Workshop on Mobile Computer Systems and Applications (WMCSA 1999), New Orleans, Louisiana, USA, February 1999, pp. 90-100 (1999)

29. Srinivasa, S., Haenggi, M.: Modeling interference in finite uniformly random networks. In: International Workshop on Information Theory for Sensor Networks, WITS 2007 (2007) 